DOI 10.31489/2020No2/110-115

UDC 621.2.082.18

\title{
STATIC INTERACTION OF ROUGH SURFACES UNDER NORMAL FORCE
}

\author{
Khairaliyev S. ${ }^{1 *}$, Kaishubayeva N. ${ }^{2}$, Spitas Ch. $^{3}$, Dzhundibayev V. ${ }^{4}$ \\ 1Intekko Ltd, Nur-Sultan, Kazakhstan, kh.serik@gmail.com \\ ${ }^{2}$ First Wind Power Station, Nur-Sultan, Kazakhstan \\ ${ }^{3}$ Nazarbayev University, School of Engineering, Nur-Sultan, Kazakhstan \\ ${ }^{4}$ L.N. Gumilyov Eurasian National University, Nur-Sultan, Kazakhstan
}

\begin{abstract}
Modern mechanical engineering designs (and some others) make more and more demands on the development of mathematical models of units in which friction is present. A condition that significantly complicates the study of the properties of a frictional contact is its discreteness with different geometric parameters of two contacting bodies, as a result of which the contact is formed only between a part of the roughness of the rubbing bodies (base and slide). The geometry of the contacting surfaces can most accurately be described by random functions, however, methods for calculating friction nodes based on random functions are very complicated. This work is the initial stage of building a model of contact interaction of two rough bodies with roughness parameters that are different for the two bodies, but constant for each individual body. In addition, the model of contact interaction is considered with a variable force compressing the contacting bodies, in the absence of an external shear force in the contact plane, and this work allows you to check the accuracy of constructing a model of interaction of friction surfaces with a relatively simple scheme of interaction of these surfaces.
\end{abstract}

Keywords: Rough surface; analytical modelling: static interaction of rough surfaces.

\section{Introduction}

At present, reliability, efficiency and environmental friendliness of machines, instruments and devices are increasingly determined by the tribological characteristics of parts and components that work with friction. Frictional resistance absorbs around 20 per cent of world energy consumption [1]. This fact draws interest of engineers and researchers towards tribological phenomena in the design and operation of machines and mechanisms.

In fact, the influence of various phenomenological aspects of friction is still quite challenging to assess and model [2]. It could include macro-, micro- and nanoscale effects such as influence of impurities, adhesion, elastic and plastic deformation and cold welding [2-13]. Descriptions of the various friction phenomena was attempted in numerous studies by considering the surface roughness effect. Surface topography observed in microscopic scale was proved to be extremely rough and of random nature, composed of alternating ledges and hollows of various dimensions referred to as surface heights, or asperities and micro cavities.

A significant contribution to the development of the adhesion-deformation theory of friction was made by I.V. Kragelskii [14], [15] and others in the form of a molecular-mechanical theory. According to this theory, friction is caused both by overcoming the forces of molecular interaction between the contacting surfaces and by the shape change of the relief of the contacting bodies as a result of elastic and plastic deformations of their surface layers. I.V. Kragelskii put forward the idea of the dual (molecular and mechanical) nature of friction. To implement external friction, shear deformations must be concentrated in the thinnest surface layers of rubbing bodies, and these layers must have lower shear strength than the base metal (the rule of a positive gradient of shear resistance).

In this paper, the contact of two rough bodies is considered and the horizontal and vertical forces of interaction are calculated, which are the sums of the set of forces of contact interaction of the roughness of two surfaces; in this work, the horizontal external force is not considered and therefore the sum / integral of the horizontal internal forces arising in each contact should be equal to zero. 


\section{Normal force model}

We will consider the contact of two surfaces at relative rest. As microscopic imaging shows, when two macroscopically flat bodies with microroughness come in contact, the contact occurs at multiple asperities of arbitrary shapes, and varying sizes and heights. The first step in the mechanical contacts analysis is the surface characterization. The knowledge of some statistical surface parameters is very important for the modeling of the surface interaction. As specified in the ASME standards, surface could be defined by waviness and roughness profiles (see Fig. 1) [16]. The first pattern is a real rough surface profile, the second can be the result of imperfect processing and vibrations, the third is the microroughness inherent in the production of the material.
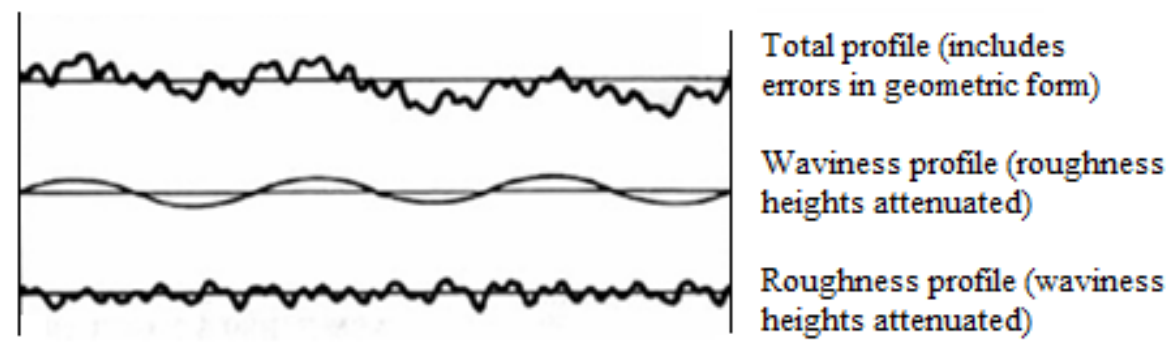

Fig. 1. Schematic diagram of surface characteristics

A detailed description of the surface microgeometry of contact bodies, for the purpose of calculating the contact interaction forces, is not practical or possible; instead a more abstract model description will be used. The main goal of the study is to describe the qualitative aspects of the processes and laws of the formation of frictional forces. For the purposes of this study, the model shown in Fig. 2 can be used, and, as shown below, this scheme allows modeling the basic principles of contact interaction forces.

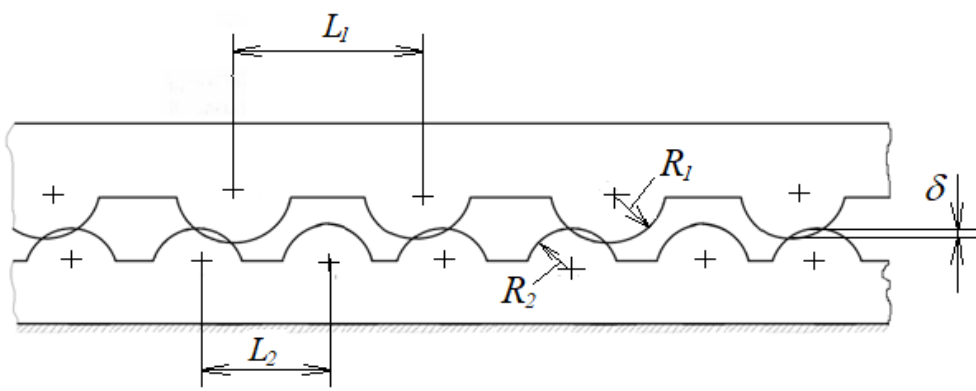

Fig. 2. Two rough contact surfaces (the slider and the base). $R_{1}$ and $R_{2}$ are micro-roughness radii of the slider and the base, respectively, $\delta$ is the overlapping of the pair of contacting micro-asperities in the vertical direction, $L_{l}$ and $L_{2}$ are period of micro-roughness of the slider and the base.

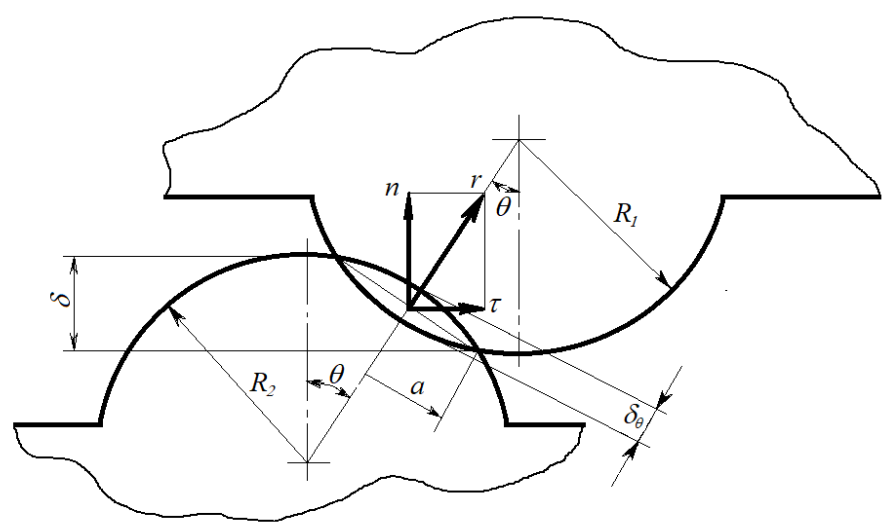

Fig. 3. Mechanical contact model between two asperities 
In Fig. 3 the following designations are adopted: $r$ is the contact reaction along the line connecting the centers of the radii of the slide and the base, $n$ and $\delta_{\theta}$ are the projections of the force of roughness interaction on the normal and tangent directions, $a$ is the radius of the contact circle of two microroughnesses, $\theta$ is the angle between the vertical and the line connecting the centers.

The theory of contact between elastic bodies or Hertz Theory can be used to compute the contact of two spherical micro-asperities. The area of contact between two spheres of radii $R_{l}$ and $R_{2}$ is the circle of radius:

where

$$
a=\sqrt{R \delta_{\theta}},
$$

$$
\frac{1}{R}=\frac{1}{R_{1}}+\frac{1}{R_{2}}
$$

The distribution of normal force in the contact area as a function of distance from the center of the circle $r$ is:

$$
p(r)=p_{0}\left(1-\frac{r^{2}}{a^{2}}\right)^{1 / 2},
$$

where: $p_{0}$ is the maximum contact pressure given by

$$
p_{0}=\frac{3 f}{2 \pi a^{2}}=\frac{1}{\pi}\left(\frac{6 f E_{*}^{2}}{R_{2}}\right)^{1 / 3} \text {, }
$$

Where $f$ is the total force of contact interaction between two micro-asperities and

$\frac{1}{E_{*}}=\frac{1-v_{1}^{2}}{E_{1}}+\frac{1-v_{2}^{2}}{E_{2}}$.

Here $E_{1}$ and $E_{2}$ are the elastic modules and $v_{1}, v_{2}$ are the Poisson's ratios associated with each body.

The area of contact is related to the applied load $f$ by the equation

$$
a^{3}=\frac{3 f R}{4 E_{*}} \text {. }
$$

The depth of indentation of two spheres (or "overlapping") $\delta_{\theta}$ can be calculated as

$\delta_{\theta}=\frac{a^{2}}{R}=\left(\frac{9 f^{2}}{16 R E_{*}^{2}}\right)^{1 / 3}$.

Hence

$f=c \delta_{\theta}{ }^{3 / 2}$

where:

$c=\frac{4}{3} R^{1 / 2} E_{*}$.

The following equation can be compiled from consideration of Fig. 3:

$R_{1}+R_{2}-\delta=\left(R_{1}+R_{2}-\delta_{\theta}\right) \cos \theta$,

and hence:

$\delta_{\theta}=\frac{\delta-\left(R_{1}+R_{2}\right)(1-\cos \theta)}{\cos \theta}$

Substitution of (2) into (1) gives:

$f=c \frac{\left[\delta-\left(R_{1}+R_{2}\right)(1-\cos \theta]^{3 / 2}\right.}{\cos \theta^{3 / 2}}$

Current study focuses on the relative sliding of two bodies (as in brakes, clutches, etc.), therefore the friction surfaces of such bodies can be considered as "lapped" and the angle $\theta$ cannot be big.

Hence $\cos \theta \approx 1,1-\cos \theta \approx \frac{\theta^{2}}{2}$ and that simplifies (3):

$f=c\left[\delta-\left(R_{1}+R_{2}\right) \frac{\theta^{2}}{2}\right]^{3 / 2}$

The use of the following symbol

gives

$$
\theta_{0}^{2}=\frac{2 \delta}{R_{1}+R_{2}}
$$

$f=c_{\theta}\left(\theta_{0}^{2}-\theta^{2}\right)^{3 / 2}$

where

$$
c_{\theta}=c\left(\frac{R_{1}+R_{2}}{2}\right)^{3 / 2} .
$$


The simplest way to compute the total force of contact interaction is the integration of (5) over the whole angle $\theta$ with apparent assumption of equal probability of any of its values from the interval

$-\theta_{L} \leq \theta \leq \theta_{L}$,

where $\theta_{L}$ is the greatest angle up to which it is assumed that the micro-asperity of the slider opposed to the micro-asperity of the base:

$$
\theta_{L}=\frac{L_{1}+L_{2}}{2\left(R_{1}+R_{2}\right)},
$$

The total force of contact interaction is

$F=\sum_{i} f_{i}$,

where the summation of forces of contact interaction is over all pairs of contacting micro-asperities. If we suppose that the number of pair of micro-asperities is big enough then the total force of contact interaction can be calculated by integrating as follows:

$$
F=\frac{j_{a}}{2 \theta_{L}} \int_{-\theta_{0}}^{\theta_{0}} f d \theta
$$

where $j_{a}$ is the number of opposing pairs of micro-asperities, $\theta_{0}$ is the angle at which the surface roughness only touch each other and it can be found as:

$$
\theta_{0}=\sqrt{\frac{2 \delta}{R_{1}+R_{2}}} \text {. }
$$

Comparison of (6) and (8) tells that for real surfaces $\theta_{0} \ll \theta_{L}$ and, therefore, only a small fraction of the total number of micro-asperities is in contact. The geometry of the frictional contact can be represented in a simplified form using Fig. 4. Here, all micro-asperities of the base are reduced to one spherical element, and a plurality of micro- asperities of the slider is presented by the gray mass. In contact all micro-roughnesses of the slider are in the range of angle from $-\theta$ to $\theta$. It should be emphasized that for relative rest of the friction pair elements, the angle to which contact exists on both sides of the slider microsphere is the same and is equal to $\pm \theta$.

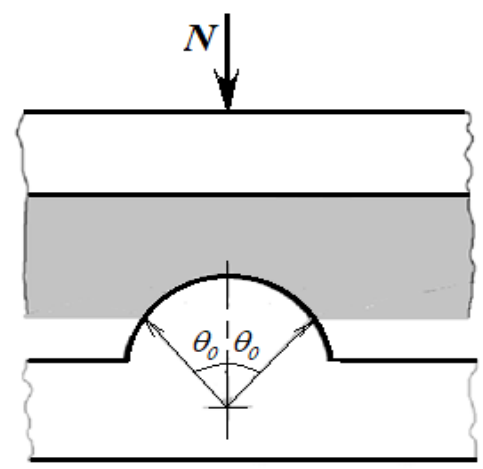

Fig. 4. Reduced model, where all microasperities of the base are reduced to one spherical.

$N$ is a vertical force applied to the slider, $\theta_{0}$ is maximum contact angle of the slider and base roughness.

The vertical component of $f$ (normal contact reaction in a single contact under angle $\theta$ to the vertical) and horizontal component (friction force) can be calculated as follows:

$$
n=f \cos \theta, \tau=f \sin \theta \text {. }
$$

As the angle $\theta$ is small, the

$$
\begin{aligned}
& \quad n \approx f\left(1-\frac{\theta^{2}}{2}\right) \approx f, \tau \approx f \theta, \\
& \text { or: } \\
& \quad n=c_{\theta}\left(\theta_{0}^{2}-\theta^{2}\right)^{3 / 2}, \tau=c_{\theta}\left(\theta_{0}^{2}-\theta^{2}\right)^{3 / 2} \theta .
\end{aligned}
$$

The total value of these forces for all of the contact points may be computed by integrating:

$$
N=\frac{j_{a} c_{\theta}}{2 \theta_{L}} \int_{-\theta_{0}}^{\theta_{0}}\left(\theta_{0}^{2}-\theta^{2}\right)^{3 / 2} d \theta,
$$




$$
T=\frac{j_{a} c_{\theta}}{2 \theta_{L}} \int_{-\theta_{0}}^{\theta_{0}}\left(\theta_{0}^{2}-\theta^{2}\right)^{3 / 2} \theta d \theta .
$$

Taking integrals (10) we finally get:

$$
\begin{aligned}
& N=\frac{3 j_{a} c_{\theta} \pi \theta_{0}^{4}}{16 \theta_{L}}, \\
& T=0 .
\end{aligned}
$$

In this system there was no external perturbation applied in the horizontal direction and the friction force $T$ equal to zero confirms the correctness of computations above. Equation (11) allows getting a formula for contact stiffness:

$$
\delta=\sqrt{\frac{\sqrt{2}\left(R_{1}+R_{2}\right)\left(L_{1}+L_{2}\right) N}{j_{a} R_{1} R_{2} E_{*}}}
$$

The vertical force of contact interaction obtained here (11) is not absolutely accurate. However, this force allows you to see the qualitative side of the contact interaction of rough surfaces and, at the same time, with a qualitative choice of the characteristics of the friction surfaces, it should allow calculating the contact interaction and friction forces with not very large errors.

\section{Results and discussion}

The vertical force of contact interaction (11) is not absolutely accurate. However, this force allows to see the qualitative side of the contact interaction of rough surfaces and, at the same time, with a qualitative choice of the characteristics of the friction surfaces, it should allow calculating the contact interaction and friction forces with not very large errors. The graph of the contact stiffness having a quadratic parabola in Fig. 5 is based on (13). More accurate theories built using more accurate description of the micro-geometry of surfaces using random functions for the distribution of the surface textures [14], [15], [17] give other power functions in these dependences. However as is evident from (13) obtained functions quite accurately reflect qualitative dependences of the processes in contacts and hence can be used to study the qualitative aspects of the friction processes.

The parameters of the friction system used to construct the graphs shown in Fig. 5 were chosen for the materials considered in [14], [15], [17]. It should be noted that the qualitative aspect of the contact stiffness graphs shown in Fig. 5 has been correspond to the real data used in these books. Our experience in calculations says that in the case of developing a sufficiently high-quality method for choosing the parameters of rough bodies, there is reason to hope for an acceptable quantitative agreement of the calculations with experimental data.

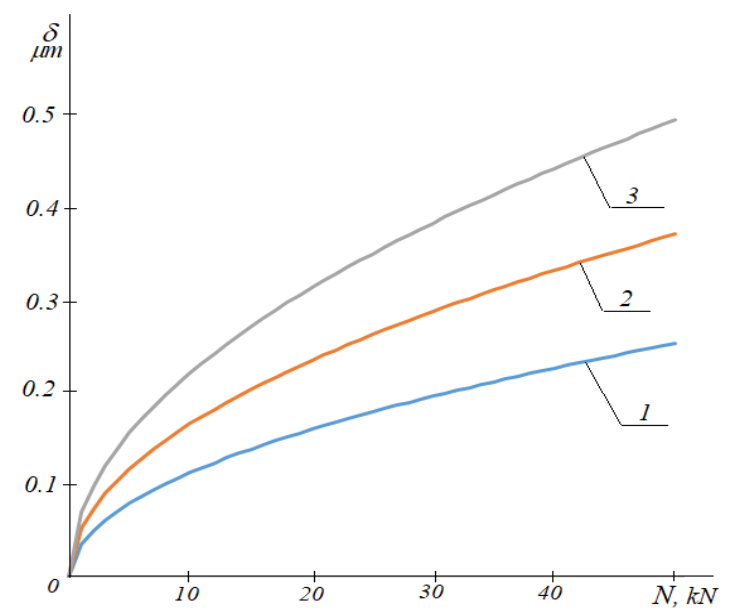

Fig. 5. Graph of contact stiffness (due to the deformation of the micro-roughness) 
Table 1. The parameters of the graphs in Fig. 5

\begin{tabular}{c|c|c|c}
\hline Lines on the graph & 1 & 2 & 3 \\
\hline$R_{l}, \mathrm{~m}$ & $5.00 \mathrm{E}-05$ & $1.50 \mathrm{E}-04$ & $3.00 \mathrm{E}-04$ \\
\hline$R_{2}, \mathrm{~m}$ & $8.00 \mathrm{E}-05$ & $2.00 \mathrm{E}-04$ & $5.00 \mathrm{E}-04$ \\
\hline$L_{1}, \mathrm{~m}$ & $1.00 \mathrm{E}-04$ & $3.00 \mathrm{E}-04$ & $5.00 \mathrm{E}-04$ \\
\hline$L_{2}, \mathrm{~m}$ & $2.00 \mathrm{E}-04$ & $6.00 \mathrm{E}-04$ & $9.00 \mathrm{E}-04$ \\
\hline$E^{*}, \mathrm{~Pa}$ & $1.067 \mathrm{E}+11$ & $1.067 \mathrm{E}+11$ & $1.067 \mathrm{E}+11$ \\
\hline$j_{a}$ & $1.00 \mathrm{E}+08$ & $5.00 \mathrm{E}+07$ & $2.00 \mathrm{E}+07$ \\
\hline
\end{tabular}

Thus, the friction pair model considered here can be used to build a qualitative model of the friction force.

\section{Conclusion}

This article deals with the static contact of rough surfaces under the action of a normal force on them, but without the action on the considered bodies of a shear force in the contact plane. The consideration of this problem includes the main parameters of the roughness of the contacting surfaces and the mechanical characteristics of the bodies and, of course, the friction force in the conditions under consideration is zero. This condition is verified by integrating the friction forces over all active contacts, and the results obtained here make it possible to accept the considered model for calculating the friction force with stable sliding of bodies relative to each other.

\section{REFERENCES}

1 Universiteit van Amsterdam, ScienceDaily, 2018. Friction: Da Vinci revisited. Available at: www.sciencedaily.com/releases/2018/03/180301091836.htm.

2 Popov, V. L., Psakhie, S. G. Numerical simulation methods in tribology. Tribol. Int., 2007, No. 40(6), pp. $916-$ 923. DOI: $10.1016 /$ j.triboint.2006.02.020

3 Savencu, O. Simulations of Dry Friction between Rough Surfaces and Corresponding Nonlinear Problems at Nano and Microscales. Ph.D. thesis, Cardiff University, Cardiff. 2016, pp. 4-6.

4 Bowden, F. P., Tabor, D. The Area of Contact between Stationary and between Moving Surfaces. Proceedings of the Royal Society of London. Series A, Mathematical and Physical Sciences, 1939, Vol. 169, Is. 938, pp. 391-413.

5 Bowden, F. P., Tabor, D. The Friction and Lubrication of Solids. Oxford: Clarendon Press. 1950, pp. 2-3.

6 Kendall, K. Molecular Adhesion and Its Applications. The Sticky Universe, Kluwer Academic Publishers. 2001, p.7-8.

7 Archard, J. F. Elastic Deformation and the Laws of Friction. Proceedings of the Royal Society of London. 1957, Vol. 243, pp. 190-205. DOI: 10.1098/rspa.1957.0214

8 Johnson, K. L., Kendall, K., Roberts, A.D. Surface Energy and the Contact of Elastic Solids. Proceedings of the Royal Society of London. Series A, Mathematical and Physical Sciences. 1971, Vol. 324, Is. 1558, pp. 301-313. DOI: 10.1098/rspa.1971.0141

9 Derjaguin, B. V., Muller, V. M., Toporov, Y. P. Effect of contact deformations on the adhesion of particles. J.Colloid Interface Sci. 1975, Vol. 53(2), pp. 314-326. DOI: 10.1016/0021-9797(75)90018-1

10 Ogilvy, J. A. Numerical simulation of friction between contacting rough surfaces J. Phys. D. Appl. Phys., 1991, Vol. 24(11), pp. 2098-2109. DOI: 10.1088/0022-3727/24/11/030

11 Maugis, D. Adhesion of spheres: The JKR-DMT transition using a dugdale model. J. Colloid Interface Sci., 1992, Vol. 150(1), pp. 243-269. DOI: 10.1016/0021-9797(92)90285-T

12 McClelland, G. M., Glosli, J. N. Friction at the Atomic Scale. in Fundamentals of Friction: Macroscopic and Microscopic Processes. NATO ASI Series (Series E: Applied Sciences), Dordrecht: Spnriger. 1992, 405-425.

13 Tabor, D. Friction. The Present State of Our Understanding. J. Lubr. Technol., 1981, Vol. 103(2), pp. 169-179. DOI: $10.1115 / 1.3251622$

14 Kragelskii, I. V., Dobychin, M. N., and Kombalov V. S. Friction and Wear. Calculation methods. Oxford: Pergamon Press Ltd. 1982, 474 p.

15 Kragelskii I.V. Friction and Wear, Elmsford: Pergamon Press, 1982, 420 p.

16 ASME B46.1-2002, 2003, "Surface Texture (Surface Roughness, Waviness, and Lay: An American National Standard," New York: American Society of Mechanical Engineers.

17 Chichinadze A.V., et al. Basics of tribology, Moscow, Machinostroenie. 2001, 660 p. 